\title{
Ocriplasmin for symptomatic vitreomacular adhesion: an evidence-based review of its potential
}

This article was published in the following Dove Press journal:

Core Evidence

21 March 2014

Number of times this article has been viewed

\author{
Su Jeong Song ${ }^{1,2}$ \\ William E Smiddy' \\ 'Department of Ophthalmology, \\ Bascom Palmer Eye Institute, \\ University of Miami Miller School \\ of Medicine, Miami, FL, USA; \\ ${ }^{2}$ Department of Ophthalmology, \\ Kangbuk Samsung Hospital, \\ Sungkyunkwan University School \\ of Medicine, Seoul, South Korea
}

Correspondence: William E Smiddy Bascom Palmer Eye Institute, 900 NW 17th Street, Miami, FL 33I36, USA

Tel +l 3053266172

Fax +I 3053266417

Email wsmiddy@med.miami.edu

\begin{abstract}
Vitreomacular traction is a multicategory entity that may cause substantial visual loss due to the formation of a macular hole or traction-induced tissue distortion. The advent of optical coherent tomography (OCT) has demonstrated the anatomic features of persistent vitreomacular attachment (VMA) more definitively, including in many asymptomatic or minimally symptomatic patients. The indications for intervention are unclear, since it is not possible to predict which eyes might be likely to develop progressive visual loss. This has been especially important since for many years, the only treatment option involved surgical intervention (vitrectomy) to release the persistent VMA. Recently, a pharmacolytic agent, ocriplasmin, has become available after many years of development and investigation, and may offer a feasible alternative to surgery, or even a risk/benefit ratio sufficiently favorable to offer intervention at an earlier stage of VMA. Several studies, including a large, prospective clinical trial, have established the foundation of its rationale and efficacy, providing the basis of its approval. The role for ocriplasmin in clinical practice is in the process of being determined. This paper summarizes current knowledge and status of investigations regarding ocriplasmin-induced pharmacologic vitreolysis, and offers some evidence-based considerations for its use.
\end{abstract}

Keywords: macular edema, microplasmin, pharmacologic vitreolysis, posterior vitreous detachment

\section{Core evidence clinical impact summary for ocriplasmin}

\begin{tabular}{|c|c|c|}
\hline Outcome measure & Evidence & Implications \\
\hline \multicolumn{3}{|l|}{ Disease-oriented evidence } \\
\hline $\begin{array}{l}\text { Vitreomacular attachment (VMA) is lysed in } \\
\text { many circumstances }\end{array}$ & $\begin{array}{l}21-23,44 \\
54,56,66\end{array}$ & $\begin{array}{l}\text { Potential role in other } \\
\text { disorders }\end{array}$ \\
\hline \multicolumn{3}{|l|}{ With higher frequency than natural history } \\
\hline \multicolumn{3}{|l|}{ Patient-oriented evidence } \\
\hline Macular hole with VMA & MIVI-TRUST ${ }^{65}$ & $\begin{array}{l}\text { A nonsurgical alternative, } \\
\text { albeit less successful }\end{array}$ \\
\hline VMA causing $20 / 25$ or worse vision & MIVI-TRUST 65 & $\begin{array}{l}\text { A nonsurgical alternative, } \\
\text { albeit less successful }\end{array}$ \\
\hline \multicolumn{3}{|l|}{ Economic evidence } \\
\hline Avoiding surgery, lower costs & $\begin{array}{l}\text { Presumed; } \\
\text { literature lacking }\end{array}$ & $\begin{array}{l}\text { May or may not lower } \\
\text { costs }\end{array}$ \\
\hline
\end{tabular}

\section{Introduction}

There is a broad spectrum of conditions that involve some degree of vitreomacular attachment that may or may not be pathogenic. At one end of the spectrum is the asymptomatic partial separation of vitreous that probably represents an early phase of 
posterior vitreous detachment (PVD) ${ }^{1}$ and is only detectable by optical coherence tomography (OCT). At the most extreme end of the spectrum is vitreous attached to the edge of a full-thickness macular hole, a configuration that invokes the hypothesis that vitreous traction overwhelmed the tensile strength of the retina to cause the hole. ${ }^{2}$ Morphologies with vitreous attachment at the macula associated with loss of the foveal depression, tenting up of the inner retinal surface, intraretinal edema, or even subretinal fluid appear to form a continuum of intermediate forms. The severity of associated symptoms generally correlates with the magnitude of these apparent traction-induced effects, specifically as they impact the outer retinal layers.

There is no consensus regarding the categorization and terminology of this family of conditions, or what might be considered its close cousins - vitreomacular traction syndrome (VMTS) and epiretinal membrane (ERM). Generally, VMTS is a more prominent form of vitreomacular attachment usually associated with a broader zone of attachment and a moderate degree of epiretinal membrane formation. It has distinct features recognizable on clinical examination and VMTS does not lead directly to macular hole formation. ${ }^{3,4}$ It may well be a more entrenched form of vitreomacular adherence, populated by secondary epiretinal membranes. ERM is commonly present in an eye that has already had a complete PVD, but might have its etiology in some vitreomacular traction stimulus that has expired. ERM and VMTS will not be further described in this review.

The advent of ocular coherence tomography (OCT) has given clarity to the vitreomacular anatomy which has allowed a much higher degree of standardization and definition of certain subgroups. The more subtle, focal, usually not clinically observable, form of vitreomacular attachment has come to be referred to as its own diagnostic subset under the moniker of vitreomacular attachment (VMA), albeit more specifically applied than in the generic way discussed above. This leaves some confusion in discussing this subset of patients. The microplasmin intravitreal injection for nonsurgical treatment of focal vitreomacular adhesion (MIVI-TRUST) study investigators have coined this more specific use of VMA in recent studies designed to test the therapeutic value of ocriplasmin. To make nomenclature matters even more confusing, those investigators have lumped macular holes with persistent vitreous attachment into that same diagnostic group as VMA.

Various factors might influence the formation or influence the course or severity of VMA; these might include vitreous liquefaction, focal anatomic factors that might potentiate foveal adhesion (for example inflammation or constitutional factors as yet undefined), or internal limiting membrane (ILM) integrity. The common denominator in all of these cases is that the VMA seems to take an active (as opposed to passive, incidental) role, transferring traction at the fovea and, in some cases, leading to progressive anatomic and symptomatic changes.

The therapeutic rationale in VMA is to release the vitreomacular attachment with the hope that anatomic and visual improvement (or at least arrested progression) results. Hitherto, surgical release has been the only option, so only more advanced VMA cases have been treated, but with respectable visual results. ${ }^{4-8}$ However, many (if not most) cases with VMA, especially those with minimal or no symptoms, may not progress to cause visual loss, and are safely observed without surgical intervention. ${ }^{9}$ Clinical intuition has restricted vitrectomy to the more severe or unequivocally progressing cases. Consequently, a group of intermediately affected eyes might be at risk of visual loss, but are not considered for treatment. The possibility of avoiding vitrectomy or of offering a therapeutic option short of vitrectomy for the group of intermediate severity has been a main focus of attempts to develop a nonsurgical therapeutic option.

The culmination of many years of investigation has been the recent approval of ocriplasmin (sometimes referred to as microplasmin), an agent that has lytic properties at the vitreoretinal interface. ${ }^{10-15}$ Even if the anatomic success rate of a less invasive therapy might be lower than vitrectomy, it might offer the appeal of avoiding surgery in some patients or expanding the group of patients who might be amenable to treatment.

The OCT finding of VMA in higher association with other conditions such as diabetic macular edema (DME) and agerelated macular degeneration (AMD) raises the hypothesis that VMA may play a pathogenic role in those conditions and hence, be amenable to therapeutic release. ${ }^{16-20}$ These hypothetical possibilities are currently being considered by many individuals, as the consensus role for ocriplasmin is being determined. ${ }^{21-24}$

This paper will review the current status of studies which may provide evidence for ocriplasmin treatment of VMA.

\section{Background studies of surgical treatment of VMA}

Impending macular holes and, subsequently, stage II or III macular holes represented the first subgroups that were operated on with the rationale of relieving the causative VMA. Initial clinical reports of vitrectomy (PPV) for impending macular holes were encouraging ${ }^{4,25-27}$ and led to a prospective, randomized, controlled clinical trial. ${ }^{28}$ The results of that trial were tempered due to the recruitment not being 
sufficient to give the study its necessary statistical power. The success in treating full thickness macular holes (especially small, early-stage ones) that occurred at that time displaced attention from the impending macular hole group to the full-thickness macular hole group, since the diagnosis and prognosis of impending macular holes was imprecise, as described above.

\section{Nonsurgical vitreolysis}

The concept of nonsurgical vitreolysis was first reported clinically by Chan who showed pneumatic vitreolysis in eyes with early macular hole formation can result in closure of the macular hole in many cases. ${ }^{29}$ This maneuver did not gain much popularity, possibly due to the high rate of success of PPV that became apparent contemporaneously. More recently, this possibility has been revisited. ${ }^{29-34}$ Rodriguez showed that intravitreal gas injection induced complete resolution of VMA in $40 \%$ of eyes by 1 month and in $60 \%$ of eyes within 6 months. ${ }^{30}$ The overall results may be equivalent to those of clinical trials of ocriplasmin (see below), but one must consider the limitations of that study - small sample size and retrospective nature. The frequency of complications, side effects, and constraints of long-acting gas and prone positioning have not been definitively quantitated, but are not negligible.

\section{Pharmacologic vitreolysis and ocriplasmin}

As the capabilities of surgery for vitreomacular traction disorders have become defined, and with the broad facility and acceptance of intravitreal injections for retinal vascular conditions, the search to find pharmacologic agents to lyse vitreoretinal attachments has been pursued with renewed interest. This approach was first conceived of for pediatric cases requiring surgery - a group in which inducing a PVD is notoriously difficult - well before VMA cases were even defined, much less considered for therapeutic intervention. Because VMA represents a focal, well-defined area of pathologic adherence, efforts to develop a pharmacologic agent targeted the molecular components of the vitreomacular interface like fibronectin and laminin. ${ }^{35,36}$

Pharmacologic vitreolytic agents can be divided into two categories: enzymatic and non-enzymatic agents. ${ }^{37}$ Enzymatic pharmacologic agents include interfactants (dispase), liquefactants (hyaluronidase, collagenase), and agents that combine both properties (plasmin, microplasmin, chondroitinase). ${ }^{37}$

Plasmin is a serine protease that directly lyses fibrin, laminin, and fibronectin, and increases levels of other proteases that disrupt extracellular matrix structures. ${ }^{38,39}$ However, plasmin is extremely unstable and although autologous plasmin is more stable, it is time consuming to use and expensive to produce. Ocriplasmin (ThromboGenics NV, Leuven, Belgium) is a recombinant human protein which contains plasmin's catalytic domain, and thus, shares the catalytic properties of human plasmin. ${ }^{40}$ Ocriplasmin's effects are relatively specific for vitreous and less active on other ocular laminin- and fibronectin-containing structures such as lens, ciliary body, retinal vessels, and lamina cribrosa. Examination of the ciliary body and zonules adjacent to the site of injection on scanning electromicroscope did not disclose any significant abnormality. ${ }^{39,41}$ Ocriplasmin is more stable than autologous plasmin, has increased sterility, and is one-fourth the size of plasmin (22 $\mathrm{kDa}$ versus $88 \mathrm{kDa})$, which likely facilitates greater penetration of vitreous and epiretinal tissues. ${ }^{41}$

The earliest in vivo studies testing the concept demonstrated that an intravitreal injection of ocriplasmin induced complete PVD in a dose- and time-dependent fashion without outer retina damage. ${ }^{38,39,42-44}$ Chen reported that $0.5 \mathrm{U}, 1.0 \mathrm{U}$, and $1.5 \mathrm{U}$ recombinant microplasminogen induced a complete PVD in $25 \%, 75 \%$, and $87.5 \%$ of rabbit eyes within 1 day. ${ }^{11}$ The concentration of recombinant microplasminogen correlates inversely to the degree of residual posterior vitreous cortical at 1 day after injection. Eyes that received $0.5 \mathrm{U}$ of recombinant microplasminogen had much more residual collagen fibrils than those that received $1.5 \mathrm{U}$.

There are a couple of clinical concerns that have not been fully vetted against the benefits of ocriplasmin. A transient decrease of the $a$ and $b$ waves of electroretinogram (ERG) was noted in all doses of ocriplasmin, but without permanent ERG or retinal ultrastructural abnormalities at doses less than $250 \mu \mathrm{g} .{ }^{41}$ The package insert of ocriplasmin (Jetrea, Thrombogenics, Leuven, Belgium)) also reports a $2 \%$ incidence of dyschromatopsia with ERG changes and a dislocated crystalline lens in a human receiving a dose $40 \%$ over the recommended and in three species (including 100\% of primates) receiving multiple doses. $^{42}$

\section{Clinical results and ongoing clinical trials of ocriplasmin}

Although ocriplasmin was first developed for the treatment of acute ischemic stroke, there are no active clinical trials of ocriplasmin besides its use in VMA-related conditions. Ocriplasmin is the only vitreolytic agent approved for clinical use to treat VMA, and VMA with or without macular 
hole is its only approved use. The scope of its use is in the process of being translated from the most prominent clinical trial results to general practice. Hence, it is worthwhile to review all of the available information regarding results and ongoing studies of ocriplasmin. There are 14 ocriplasmin trials listed in the registry of clinical trials (www.clinicaltrials.gov). The majority have been completed and are summarized below. The title listed on the registry is presented in boldface, but in most instances the protocol title within the study heading is worded differently and is presented here as a sub title.

\section{Microplasmin intravitreal administration in} patients with vitreomacular traction scheduled for vitrectomy

(Intravitreal microplasmin in patients undergoing surgical vitrectomy, MIVI-I) ${ }^{2 I}$

MIVI-I was a Phase IIa safety study to assess the safety of recombinant ocriplasmin intravitreal administration and to obtain an early indication of possible efficacy. Sixty patients in six cohorts (ten patients each) received varied doses (25-125 $\mu \mathrm{g}$ ) or different exposure time (1 hour-7 days). All patients had been scheduled for PPV due to VMA associated with VMT, diabetic macular edema, or a stage II-III macular hole of less than 6 months' duration. The outcome endpoint was PVD determined at the time of vitrectomy. The use of ocriplasmin led to a progressively higher incidence of PVD in $0,10 \%$, and $25 \%$ of patients with increasing time exposures ( 2 hours, 24 hours, 7 days). These results demonstrated the safety of intravitreal ocriplasmin and confirmed that ocriplasmin could induce PVD in some patients.

Intravitreal injection of microplasmin for treatment of vitreomacular adhesion: results of a prospective, randomized, sham-controlled Phase II trial

(A study to compare multiple doses intravitreal of microplasmin or treatment of patients with vitreomacular traction, MIVI-IIt) ${ }^{22}$

This study was designed to evaluate the preliminary proof of concept of pharmacolytic correction of VMA. Inclusion criteria included a partial PVD as demonstrated by ultrasound examination and OCT with foveal VMA, a macular thickness of at least $250 \mu \mathrm{m}$, and best-corrected visual acuity (BCVA) of 20/40 or less in the study eye and 20/400 or better in the fellow eye. The primary endpoint was release of VMA at 28 days after injection. Sixty eligible patients were randomized into four cohorts of 15 patients each. Three of the cohorts included a 4:1 randomization (12 treated, three controls), one cohort for each dose: $75 \mu \mathrm{g}, 125 \mu \mathrm{g}$, or $175 \mu \mathrm{g}$ ocriplasmin or sham treatment. The primary clinical endpoint, nonsurgical resolution of VMA 28 days after injection, was observed in one $(8 \%)$ patient in the sham cohort, three $(25 \%)$ patients in the $75 \mu \mathrm{g}$ ocriplasmin cohort, eleven (44\%) patients in the $125 \mu \mathrm{g}$ ocriplasmin cohort, and three (27\%) patients in the $175 \mu \mathrm{g}$ ocriplasmin cohort. The $125 \mu \mathrm{g}$ ocriplasmin cohort had a higher proportion resulting in total PVD compared with the sham cohort $(P=0.046)$. Total PVD in the pooled ocriplasmin treatment cohorts was more common than in the sham treatment cohort at day $90(P=0.033)$ after injection, but there was not a difference at day 180 after injection $(P=0.10)$.

All members of the fourth cohort received $125 \mu \mathrm{g}$ of intravitreal ocriplasmin or sham (4:1 randomization) monthly until the VMA was released, up to a total of three doses. In this cohort, seven of 12 ocriplasmin-treated and two of three sham-treated patients did not have resolution of VMA at the day 28 visit and received a repeat injection. There were no statistically significant differences in the proportion of patients achieving a BCVA improvement of at least 15 letters in the treatment cohorts versus the observed cohorts despite the release of the VMA.

This study indicates that intravitreal injection of ocriplasmin can result in nonsurgical resolution of VMA in 44\% of patients in the $125 \mu \mathrm{g}$ treatment group.

\section{A placebo-controlled trial of microplasmin} intravitreous injection to facilitate posterior vitreous detachment before vitrectomy

(A study of the safety and efficacy of microplasmin to induce a posterior vitreous detachment, MIVI-III) ${ }^{23}$

This Phase II, multicenter, randomized, double-masked, placebo-controlled, parallel-group trial combined features of the previous two studies. Three doses of ocriplasmin (25 $\mu \mathrm{g}, 75 \mu \mathrm{g}$, and $125 \mu \mathrm{g}$ ) were compared with placebo, but injections were administered 7 days ( \pm 1 day) before planned pars plana vitrectomy (PPV). The primary efficacy outcome was total PVD without creation of an anatomic defect (ie, retinal tear or retinal detachment) as ascertained by the surgeon's visualization at the beginning of vitrectomy before suction or any other mechanical intervention. The secondary endpoints were a gain of $\geq 1, \geq 2, \geq 3$ lines in BCVA and the number in which vitrectomy was avoided due to PVD occurrence.

The rates of total PVD at time of surgery were $10 \%, 14 \%$, $21 \%$, and $31 \%$ for the placebo, $25 \mu \mathrm{g}, 75 \mu \mathrm{g}$, and $125 \mu \mathrm{g}$ 
ocriplasmin groups, respectively. The mean BCVA letters of improvement from baseline 35 days after injection were 1.1, 3.6, 3.2, and 8.4 for the placebo, $25 \mu \mathrm{g}, 75 \mu \mathrm{g}$, and $125 \mu \mathrm{g}$ ocriplasmin groups, respectively. Comparing ocriplasmin to placebo, only the $125 \mu \mathrm{g}$ ocriplasmin group improvement was statistically significant $(P<0.05,2$-sample $t$-test $)$.

\section{Enzymatic vitreolysis with ocriplasmin for vitreomacular traction and macular holes (Trial of microplasmin intravitreal injection for nonsurgical treatment of focal vitreomacular adhesion, also known as the MIVI-TRUST [TG-MV-006 \& TG- MV-007] trial) ${ }^{24}$}

This pivotal study publication reported on two parallel, multicenter, randomized, double-blind, Phase III clinical trials. Both studies had the same study protocol except that the ratio of randomized assignments to ocriplasmin and placebo in study TG-MV-006 was 2:1, and the randomization ratio in study TG-MV-007 was 3:1. The purpose was to compare a single intravitreal injection of ocriplasmin $(125 \mu \mathrm{g})$ with a placebo saline injection in patients with symptomatic VMA. Patients with VMA alone as well as VMA with macular hole were included. The primary endpoint was resolution of VMA at day 28. Secondary endpoints were total PVD and nonsurgical closure of a macular hole at 28 days, avoidance of vitrectomy, and change in BCVA. OCT was required for study entry and for determination of the primary study endpoint.

VMA resolved in 26.5\% (123/464) of ocriplasmininjected eyes and in $10 \%(19 / 188)$ of placebo-injected eyes $(P<0.001)$. Total PVD occurred in 13.4\% (62/464) of ocriplasmin-treated eyes and 3.7\% (7/188) of placebo-treated eyes $(P<0.001)$. Nonsurgical closure of macular holes was achieved in $40.6 \%(43 / 106)$ of ocriplasmin-injected eyes, as compared with $10.6 \%(5 / 47)$ of placebo-injected eyes $(P<0.001)$. The BCVA was improved by at least three lines in $12.3 \%(57 / 464)$ of ocriplasmin-treated eyes and $6.4 \%$ $(12 / 188)$ of placebo-treated eyes $(95 \%$ confidence interval [CI]: 1.26-7.76, $P=0.02$ ). The incidence of retinal tears or detachments was similar in the two groups $(1.9 \%$ versus $4.3 \%, P=0.11$ ).

The subgroup analysis focusing on the OCT characteristics showed that presence of an epiretinal membrane decreased ocriplasmin efficacy. Among patients without an epiretinal membrane, $37.4 \%$ in the ocriplasmin group had nonsurgical resolution of VMA, as compared to $14.3 \%$ in the placebo group (odds ratio [OR]: 3.79; 95\% CI: 2.09-7.22). Among patients with an epiretinal membrane, resolution of VMA occurred in only $8.7 \%$ in the ocriplasmin group compared to $1.5 \%$ in the placebo group (OR: 6.20; $95 \%$ CI: 0.93-2.65). These findings support the hypothesis that epiretinal membranes increase the strength of the VMA by fibrocellular organization and contraction. ${ }^{43}$ Another subgroup analysis demonstrated a poor prognosis for a broader width of vitreous adhesion OCT grading reproducibility for VMA, broad versus focal width of vitreous adhesion, full thickness macular hole, and epiretinal membrane was high $($ kappa $>0.85) \cdot{ }^{43}$

A randomized, sham-injection-controlled, doublemasked, ascending-dose, dose-range-finding trial of microplasmin intravitreal injection for nonsurgical posterior vitreous detachment (PVD) induction for treatment of DME

(A multicenter study to compare multiple doses of intravitreal microplasmin versus sham injection for treatment of patients with diabetic macular edema [DME] MIVI-II) ${ }^{44}$

This study explored the possibility that pharmacolysis of VMA in patients with DME might be clinically helpful. That the vitreous may play a role in the pathogenesis of at least some cases of DME is inferred from two clinical observations: 1) DME can improve with spontaneous $\mathrm{PVD}^{17}$ and 2) the incidence of DME is lower among eyes with PVD compared to those without PVD. ${ }^{16}$ These studies suggested that purposeful removal of VMA in eyes with DME might improve traction-induced derangements of the anatomy. Since the report by Lewis ${ }^{7}$ first describing a beneficial effect of PPV with release of a thickened and taut posterior hyaloid traction on the macula, many retrospective studies have shown the advantage of vitrectomy and removal of the posterior hyaloid on morphologic and functional results. ${ }^{8,45-50}$ Some of these studies recommended that early surgical intervention may result in better visual outcome. ${ }^{7,8,45-50}$ However, the results with this approach have been mixed. Most studies have not included a nonsurgical control group, and the results have not deviated markedly from a natural history study. ${ }^{51}$ The largest multicenter trial did not find overall visual improvement. ${ }^{50}$ Hence, the rationale for pharmacologic lysis of VMA is less convincing for this subset.

Still, there might be an as yet undefined subset of eyes that might benefit from traction release surgically or pharmacologically. ${ }^{50}$ Proposed mechanisms of visual acuity improvement include avoiding reproliferation of glial cells, improving oxygen supply to the retina, and removing a vascular 
endothelial growth factor (VEGF) reservoir. ${ }^{52}$ Conversely, removal of the vitreous, shortens the intraocular half-life of anti-VEGF agents, if that is subsequently needed..$^{53}$

The Phase II multicenter study in the above subtitle compared multiple doses of intravitreal ocriplasmin for nonsurgical PVD induction for treatment of patients with DME. The primary efficacy variable was the proportion of patients with total PVD 14 days after the $25 \mu \mathrm{g}, 75 \mu \mathrm{g}$, $125 \mu \mathrm{g}$ of ocriplasmin, and sham injection. According to study results posted at clinicaltrials.gov, there were no statistically significant differences of PVD induction between $25 \mu \mathrm{g}, 75 \mu \mathrm{g}$, or $125 \mu \mathrm{g}$ versus the sham group.

A randomized, sham-injection-controlled, doublemasked, multicenter trial of ocriplasmin intravitreal injection for treatment of focal vitreomacular adhesion in subjects with exudative age-related macular degeneration

(Safety and efficacy study of intravitreal ocriplasmin in subjects with AMD with focal vitreomacular adhesion MIVI-5) $)^{54}$

This Phase II study evaluated the safety and efficacy of intravitreal ocriplasmin injection in subjects with exudative AMD with focal VMA. Some have recognized what appears to be a higher rate of persistent vitreomacular attachment among patients with exudative age-related macular degeneration (AMD). ${ }^{18,20}$ As with vitrectomy for DME, some have performed pilot studies of surgery for this subgroup without distinct benefit. Thus, the hypothesis that pharmacologic vitreolysis might be beneficial is less convincing in this subgroup also. However, this is the hypothesis tested with the above entitled study.

The primary outcome measurement of this study was the proportion of subjects with release of focal vitreomacular adhesion by day 28. This study was completed at April 2013, but no study results have been reported.

An open-label, single center trial of microplasmin intravitreal injection for nonsurgical treatment of focal vitreomacular adhesion

(Safety and efficacy study of microplasmin for nonsurgical treatment of focal vitreomacular adhesion MIVI-8) ${ }^{55}$

This Phase II study evaluated the safety and efficacy of a single $125 \mu \mathrm{g}$ dose of intravitreal ocriplasmin for participants with VMA. The primary outcome measure of this study was full ophthalmologic examination at baseline,
7, 14, 28 days, 3 months, and 6 months after injection. A secondary outcome measure was the proportion of patients with nonsurgical resolution of focal VMA adhesion at study visits other than the 28-day post-injection visit. This study was completed at April 2011, but no results have been reported.

A randomized, placebo-controlled, double-masked, clinical trial of intravitreal microplasmin in infants and children scheduled for vitrectomy

(Clinical trial of intravitreal microplasmin in infants and children scheduled for vitrectomy MIC) ${ }^{56}$

This study evaluated the potential benefit in inducing PVD in pediatric eyes undergoing vitrectomy not necessarily for VMA. Autologous plasma has been reported as an adjunct to pediatric vitrectomy in a variety of clinical conditions including: traumatic macular hole, stage 5 retinopathy of prematurity (ROP), and congenital X-linked retinoschisis..$^{57-62}$ In pediatric traumatic macular hole cases without PVD, after injection of ocriplasmin, a complete PVD was noted during vitrectomy in $23 \%$ and a partial PVD was noted in $15 \%$ of eyes. ${ }^{61}$

Safety was assessed through the use of reported adverse events, ophthalmic examinations, B-scan, fundus photography, and fluorescein angiography. This study was completed at April 2012, but no results have been reported.

An open-label, ascending-exposure time, single center trial to evaluate the pharmacokinetic properties of ocriplasmin intravitreal injection in subjects scheduled for primary pars plana vitrectomy

(The purpose of this study is to evaluate the pharmacokinetic properties of intravitreal ocriplasmin prior to planned primary pars plana vitrectomy [PPV] MIVI-10) $)^{63}$

This Phase II study evaluated the pharmacokinetic properties of a $125 \mu \mathrm{g}$ dose of intravitreal ocriplasmin administrated at different time-points prior to planned primary PPV. The primary outcome measure, ocriplasmin activity levels, was ascertained in vitreous samples obtained at the beginning of vitrectomy with five time-points after preoperative injection: 5-30 minutes, 31-60 minutes, 2-4 hours, 24 hours, and 7 days. The controls did not receive a preoperative injection. Thirty-eight subjects were enrolled, but two were excluded from analysis. The ocriplasmin activity levels in vitreous samples decreased from $11,597.711 \pm 7,637.41 \mathrm{ng} / \mathrm{mL}$ (5-30 minutes after 
injection) to $8,108.726 \pm 5,181.85$ (31-60 minutes),

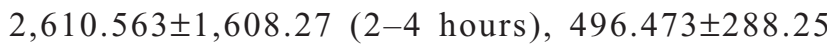
(24 hours), 272.370 (7 days). No statistical analysis has been reported.

Follow-up study to assess visual function in subset of patients who have previously participated in the TGMV-006 and TG-MV-007 ocriplasmin studies

(Follow-up study to assess visual function in subset of patients who have previously participated in the TGMV-006 and TG-MV-007 ocriplasmin studies) ${ }^{64}$

This study evaluated BCVA, spectral domain optical coherence tomography abnormality, and electroretinography abnormality in MIVI-TRUST study participants. This study was completed at October 2011, but no results have been reported.

A randomized, sham-controlled, double-masked, multicenter study evaluating ocriplasmin treatment for symptomatic vitreomacular adhesion/(VMT) including macular hole (Ocriplasmin for treatment for symptomatic vitreomacular adhesion/(VMT) including macular hole [OASIS] $)^{65}$

This Phase II study was designed to assess anatomical and functional outcomes following a single $125 \mu \mathrm{g}$ intravitreal injection of ocriplasmin in subjects with symptomatic vitreomacular adhesion including macular hole. The primary outcome measure was the proportion of subjects with pharmacological VMA resolution at day 28 after injection. This study is ongoing with an estimated completion date in 2015 .

Resolution of vitreomacular adhesion (VMA) associated with neovascular age-related macular degeneration (AMD) with intravitreal microplasmin (Study of intravitreal microplasmin in relieving vitreomacular adhesion in neovascular age-related macular degeneration $[\mathrm{AMD}])^{66}$

This Phase II study evaluated a single intravitreal injection of $1.875 \mathrm{mg}$ (high dose) ocriplasmin intravitreal injection in exudative AMD patients with VMA. The control arm consisted of one intravitreal injection of the placebo. The primary outcome is the proportion with release of VMA as assessed by ultrasonography, optical coherence tomography, and clinical examination. Secondary outcomes were total number of ranibizumab injections following ocriplasmin in eyes with
PVD versus those without PVD. No further information or results have been reported.

Microplasmin intravitreal administration in participants with uveitic macular edema

(Microplasmin intravitreal administration in participants with uveitic macular edema MIME) ${ }^{67}$

This study was sponsored by National Institute of Health Clinical Center. The participants received an intravitreal injection of $125 \mu \mathrm{g}$ of ocriplasmin at baseline. This study was terminated early due to lack of enrollment.

\section{Interaction with other drugs}

Possible interactions of ocriplasmin with other drugs, such as anti-VEGF agents, have not been evaluated. The MIVI trials have specifically excluded the eyes with a history of intravitreal injection of any drugs in the preceding 30 days. ${ }^{17}$ The hypothesis that a PVD may influence the retinal penetration or pharmacokinetics of intravitreally injected drugs is supported by animal studies, ${ }^{53,68,69}$ as has been demonstrated for antiVEGF agents. ${ }^{53}$ This hypothesis has not been confirmed (or refuted) by any clinical trial results.

\section{Conclusion}

The optimal treatment of the family of conditions characterized by vitreomacular attachments is very much an ongoing work. Patients with symptomatic, progressive VMA have formerly only had the option of treatment with PPV. Intravitreal ocriplasmin offers an alternative treatment either to avoid PPV or treat eyes at earlier stages than would generally be considered for surgery. Randomized, controlled trials have demonstrated anatomic efficacy with pharmacologic vitreolysis, but only in about $1 / 3$ of eyes. Certain subsets of patients have been identified to have a better prognosis (macular holes $<250 \mu \mathrm{m}$; no ERM; smaller length of VMA) and represent optimal candidates for ocriplasmin injection. The low rate of progression among asymptomatic or minimally symptomatic cases of VMA invokes the need for clinical judgment even for this seemingly less invasive therapy. Safety results seem satisfactory, as long as multiple or higherthan-recommended doses are avoided. Visual results have been less encouraging.

It would seem that the role for ocriplasmin should be considered only after full disclosure to patients that the success rate is low, and the agent fairly costly. Injection does not 
appear to alleviate subsequent PPV if primary success is not obtained. Caution regarding certain possible complications is advised. Perhaps only patients with progressive or substantial visual symptoms should be considered, and OCT characteristics could be confirmatory of the clinical assessment rather than provide the actual basis for timing of PPV.

The role for ocriplasmin to enhance release of vitreomacular adhesion in cases besides VMA, for example DME, AMD, and pediatric eyes before vitrectomy, has been studied, but preliminary results are not as positive as for VMA, and should still be considered experimental.

\section{Disclosure}

The authors report no other conflicts of interest in this work.

\section{References}

1. Johnson MW. Perifoveal vitreous detachment and its macular complications. Trans Am Ophthalmol Soc. 2005;103:537-567.

2. Gass JD. Reappraisal of biomicroscopic classification of stages of development of a macular hole. Am J Ophthalmol. 1995;119(6): 752-759.

3. Sonmez K, Capone A Jr, Trese MT, Williams GA. Vitreomacular traction syndrome: impact of anatomical configuration on anatomical and visual outcomes. Retina. 2008;28(9):1207-1214.

4. Margherio RR, Trese MT, Margherio AR, Cartright K. Surgical management of vitreomacular traction syndromes. Ophthalmology. 1989;96(9):1437-1445.

5. Melberg NS, Williams DS, Balles MW, et al. Vitrectomy for vitreomacular traction syndrome with macular detachment. Retina. 1995;15(3): 192-197.

6. Yamada N, Kishi S. Tomographic features and surgical outcomes of vitreomacular traction syndrome. Am J Ophthalmol. 2005;139(1): $112-117$.

7. Lewis H, Abrams GW, Blumenkranz MS, Campo RV. Vitrectomy for diabetic macular traction and edema associated with posterior hyaloidal traction. Ophthalmology. 1992;99(5):753-759.

8. Harbour JW, Smiddy WE, Flynn HW Jr, Rubsamen PE. Vitrectomy for diabetic macular edema associated with a thickened and taut posterior hyaloid membrane. Am J Ophthalmol. 1996;121(4):405-413.

9. John VJ, Flynn HW Jr, Smiddy WE, et al. Clinical course of vitreomacular adhesion managed by initial observation. Retina. 2013. Epub August 7, 2013.

10. Hermel M, Dailey W, Hartzer MK. Efficacy of plasmin, microplasmin, and streptokinase-plasmin complex for the in vitro degradation of fibronectin and laminin - implications for vitreoretinal surgery. Curr Eye Res. 2010;35(5):419-424.

11. Chen W, Mo W, Sun K, Huang X, Zhang YL, Song HY. Microplasmin degrades fibronectin and laminin at vitreoretinal interface and outer retina during enzymatic vitrectomy. Curr Eye Res. 2009;34(12):1057-1064.

12. Sazonova IY, Thomas BM, Gladysheva IP, Houng AK, Reed GL. Fibrinolysis is amplified by converting alpha-antiplasmin from a plasmin inhibitor to a substrate. J Thromb Haemost. 2007;5(10):2087-2094.

13. Elkareem GAM, Willekens B, Vanhove M, Noppen B, Stassen JM, de Smet MD. Characterization of a stabilized form of microplasmin for the induction of posterior vitreous detachment. Curr Eye Res. 2010;35(10):909-915.

14. Chen W, Huang X, Ma XW, Mo W, Wang WJ, Song HY. Enzymatic vitreolysis with recombinant microplasminogen and tissue plasminogen activator. Eye. 2008;22(2):300-307.
15. Harooni M, McMillan T, Refojo M. Efficacy and safety of enzymatic posterior vitreous detachment by intravitreal injection of hyaluronidase. Retina. 1998;18(1):16-22.

16. Nasrallah FP, Jalkh AE, Van Coppenolle F, et al. The role of the vitreous in diabetic macular edema. Ophthalmology. 1988;95(10):1335-1339.

17. Yamamoto T, Akabane N, Takeuchi S. Vitrectomy for diabetic macular edema: the role of posterior vitreous detachment and epimacular membrane. Am J Ophthalmol. 2001;132(3):369-377.

18. Krebs I, Brannath W, Glittenberg C, et al. Posterior vitreomacular adhesion: a potential risk factor for exudative age-related macular degeneration? Am J Ophthalmol. 2007;144(5):741-746.

19. Lee SJ, Lee CS, Koh HJ. Posterior vitreomacular adhesion and risk of exudative age-related macular degeneration: paired eye study. Am J Ophthalmol. 2009;147(4):621-626. e1.

20. Simpson A, Petrarca R, Jackson TL. Vitreomacular adhesion and neovascular age-related macular degeneration. Surv Opthalmol. 2012;57(6):498-509.

21. de Smet A, Gandorfer P, Stalmans P, et al. Microplasmin intravitreal administration in patients with vitreomacular traction scheduled for vitrectomy: the MIVI I trial Ophthalmology. 2009;116(7): 1349-1355.

22. Stalmans P, Delaey C, de Smet MD, et al. Intravitreal injection of microplasmin for treatment of vitreomacular adhesion: results of a prospective, randomized, sham-controlled phase II trial (the MIVI-IIT trial). Retina. 2010;30(7):1122-1127.

23. Benz MS, Packo KH, Gonzalez V, et al. A placebo-controlled trial of microplasmin intravitreous injection to facilitate posterior vitreous detachment before vitrectomy. Ophthalmology. 2010;117(4):791-797.

24. Stalmans P, Benz MS, Gandorfer A, et al; MIVI-TRUST Study Group. Enzymatic vitreolysis with ocriplasmin for vitreomacular traction and macular holes. N Engl J Med. 2012;367(7):606-615.

25. Garretson BR, Pollack JS, Ruby AJ, et al. Vitrectomy for asymptomatic lamellar macular hole. Ophthalmology. 2008;115(5):884-886.

26. Jost BF, Hutton WL, Fuller DG, et al. Vitrectomy in eyes at risk for macular hole formation. Ophthalmology. 1990;97(7):843-847.

27. Smiddy WE, Michels RG, Glaser BM, de Bustros SN. Vitrectomy for impending idiopathic macular hole. Am J Ophthalmol. 1988;105(4):371-376.

28. de Bustros S. Vitrectomy for prevention of macular holes. Results of a randomized multicenter clinical trial. Vitrectomy for Prevention of Macular Hole Study Group. Ophthalmology. 1994;101(6):1055-1059.

29. Chan CK, Wessels IF, Friedrichsen EJ. Treatment of idiopathic macular holes by induced posterior vitreous detachment. Ophthalmology. 1995;102(5):757-767.

30. Rodrigues IA, Stangos AN, McHugh DA, Jackson TL. Intravitreal injection of expansile perfluoropropane $(\mathrm{c}(3) \mathrm{f}(8))$ for the treatment of vitreomacular traction. Am J Ophthalmol. 2013;155(2):270-276. e2.

31. Johnson MW. How should we release vitreomacular traction: surgically, pharmacologically, or pneumatically? Am J Ophthalmol. 2013;155(2):203-205. e1.

32. Jorge R, Costa RA, Cardillo JA, et al. Optical coherence tomography evaluation of idiopathic macular hole treatment by gas-assisted posterior vitreous detachment. Am J Ophthalmol. 2006;142(5):869-871.

33. Mori K, Saito S, Gehlbach PL, Yoneya S. Treatment of stage 2 macular hole by intravitreous injection of expansile gas and induction of posterior vitreous detachment. Ophthalmology. 2007;114(1):127-133.

34. Ochoa-Contreras D, Delsol-Coronado L, Buitrago ME, Velasco-Barona C, Quiroz-Mercado H. Induced posterior vitreous detachment by intravitreal sulfur hexafluoride (SF6) injection in patients with nonproliferative diabetic retinopathy. Acta Ophthalmol Scand. 2000;78(6): 687-688.

35. Sebag J. Molecular biology of pharmacologic vitreolysis. Trans Am Ophthalmol Soc. 2005;103:473-494.

36. Gandorfer A. Enzymatic vitreous disruption. Eye. 2008;22(10): 1273-1277.

37. Schneider EW, Johnson MW. Emerging nonsurgical methods for the treatment of vitreomacular adhesion: a review. Clin Ophthalmol. 2011;5:1151-1165. 
38. Gandorfer A, Rohleder M, Sethi C, et al. Posterior vitreous detachment induced by microplasmin. Invest Ophthalmol Vis Sci. 2004;45(2): 641-647.

39. de Smet MD, Valmaggia C, Zarranz-Ventura J, Willekens B. Microplasmin: ex vivo characterization of its activity in porcine vitreous. Invest Ophthalmol Vis Sci. 2009;50(2):814-819.

40. Shi GY, Wu HL. Isolation and characterization of microplasminogen; a low molecular weight form of plasminogen. J Biol Chem. 1988; 263(32):17071-17075.

41. Sakuma T, Tanaka M, Mizota A, et al. Safety of in vivo pharmacologic vitreolysis with recombinant microplasmin in rabbit eyes. Invest Ophthalmol Vis Sci. 2005;46(9):3295-3299.

42. Jetrea ${ }^{\circledR}$ (ocriplasmin). [package insert]. Iselin, NJ: ThromoGenics; 2013. http://www.accessdata.fda.gov/drugsatfda_docs/label/ 2012/125422s000 lbl.pdf. Accessed September 30, 2013.

43. DeCroos FC, Toth CA, Folgar FA, et al. Characterization of vitreoretinal interface disorders using OCT in the interventional phase 3 trials of ocriplasmin. Invest Ophthalmol Vis Sci. 2012;53(10):6504-6511.

44. ThromboGenics. A multicenter study to compare multiple doses of intravitreal microplasmin versus sham injection for treatment of patients with diabetic macular edema (DME) (MIVI-II). Available from: http:// clinicaltrials.gov/show/NCT00412451. NLM identifier: NCT00412451. Accessed September 30, 2013

45. Hikichi T, Fujio N, Akiba J, et al. Association between the short-term natural history of diabetic macular edema and the vitreomacular relationship in type II diabetes mellitus. Ophthalmology. 1997;104(3): 473-478.

46. Dillinger $P$, Mester U. Vitrectomy with removal of the internal limiting membrane in chronic diabetic macular oedema. Graefes Arch Clin Exp Ophthalmol. 2004;242(8):630-637.

47. Gandorfer A, Messmer EM, Ulbig MW, Kampik A. Resolution of diabetic macular edema after surgical removal of the posterior hyaloid and the inner limiting membrane. Retina. 2000;20(2):126-133.

48. Gaucher D, Sebah C, Erginay A, et al. Optical coherence tomography features during the evolution of serous retinal detachment in patients with diabetic macular edema. Am J Ophthalmol. 2008;145(2): 289-296.

49. Gandorfer A. Role of vitreous in diabetic macular edema. Retina. 2012;32 Suppl 2:S211-S215.

50. Diabetic Retinopathy Clinical Research Network Writing Committee, Haller JA, Qin H, Apte RS, et al. Vitrectomy outcomes in eyes with diabetic macular edema and vitreomacular traction. Ophthalmology. 2010;117(6):1087-1093.

51. Parolini B, Panuzzo G. Vitrectomy for nontractional diffuse diabetic macular edema: study design and preliminary results of the VIDE Study. Ophthalmology. 2006;113(S):193.

52. Meyer CH. Current Treatment Approaches in Diabetic Macular Edema Ophthalmologica. 2007;221(2):118-131.

53. Kakinoki M, Sawada O, Sawada T, Saishin Y, Kawamura H, Ohji M. Effect of vitrectomy on aqueous VEGF concentration and pharmacokinetics of bevacizumab in macaque monkeys. Invest Ophthalmol Vis Sci. 2012;53(9):5877-5880.

54. ThromboGenics. Safety and efficacy study of intravitreal ocriplasmin in subjects with amd with focal vitreomacular adhesion (MIVI-5). Available from: http://clinicaltrials.gov/show/NCT00913744. NLM identifier: NCT00913744. Accessed September 30, 2013.

\section{Core Evidence}

\section{Publish your work in this journal}

Core Evidence is an international, peer-reviewed open-access journal evaluating the evidence underlying the potential place in therapy of drugs throughout their development lifecycle from preclinical to postlaunch. The focus of each review is to evaluate the case for a new drug or class in outcome terms in specific indications and patient groups.
55. ThromboGenics. Safety and efficacy study of microplasmin in for nonsurgical treatment of focal vitreomacular adhesion (MIVI-8). Available from: http://clinicaltrials.gov/show/NCT01055535. NLM identifier: NCT01055535. Accessed September 30, 2013.

56. ThromboGenics. clinical trial of intravitreal microplasmin in infants and children scheduled for vitrectomy (MIC). Available from: http:// clinicaltrials.gov/show/NCT00986362. NLM identifier: NCT00986362. Accessed September 30, 2013.

57. Tsukahara Y, Honda S, Imai H, et al. Autologous plasmin assisted vitrectomy for stage 5 retinopathy of prematurity: a preliminary trial. Am J Ophthalmol. 2007;144(1):139-141.

58. Wu WC, Drenser KA, Lai M, et al. Plasmin enzyme-assisted vitrectomy for primary and reoperated eyes with stage 5 retinopathy of prematurity. Retina. 2008;28:S75-S80.

59. Wu WC, Drenser KA, Capone A, et al. Plasmin enzyme assisted vitreoretinal surgery in congenital X-linked retinoschisis: surgical techniques based on a new classification system. Retina . 2007;27(8):1079-1085.

60. Wu WC, Drenser KA, Trese MT, et al. Pediatric traumatic macular hole: results of autologous plasmin enzyme-assisted vitrectomy. Am J Ophthalmol. 2007;144(5):668-672.

61. Wong SC, Capone A Jr. Microplasmin (ocriplasmin) in pediatric vitreoretinal surgery: update and review. Retina. 2013:33(2):339-348.

62. ThromboGenics. The purpose of this study is to evaluate the pharmacokinetic properties of intravitreal ocriplasmin prior to planned primary pars plana vitrectomy (PPV) (MIVI-10). Available from: http:// clinicaltrials.gov/show/NCT01159665. NLM identifier: NCT01159665. Accessed September 30, 2013.

63. ThromboGenics. Follow-up study to assess visual function in subset of patients who have previously participated in the TG-MV-006 and TG-MV-007 ocriplasmin studies. Available from: http:/clinicaltrials. gov/show/NCT01287988. NLM identifier: NCT01287988. Accessed September 30, 2013

64. ThromboGenics. Ocriplasmin for treatment for symptomatic vitreomacular adhesion/(VMT) including macular hole (OASIS). Available from: http://clinicaltrials.gov/show/NCT01429441. NLM identifier: NCT01429441. Accessed September 30, 2013.

65. University of California, Los Angeles; ThromboGenics. Study of intravitreal microplasmin in relieving vitreo-macular adhesion in neovascular age-related macular degeneration (AMD). Available from: http:// clinicaltrials.gov/show/NCT00996684. NLM identifier: NCT00996684. Accessed September 30, 2013.

66. National Institutes of Health Clinical Center (CC); National Eye Institute (NEI). Microplasmin intravitreal administration in participants with uveitic macular edema (MIME). Available from: http://clinicaltrials. gov/show/NCT01194674. NLM identifier: NCT01194674. Accessed September 30, 2013.

67. Goldenberg DT, Giblin FJ, Cheng M, et al. Posterior vitreous detachment with microplasmin alters the retinal penetration of intravitreal bevacizumab (Avastin) in rabbit eyes. Retina. 2011;31(2):393-400.

68. Sebag J, Ansari RR, Suh KI. Pharmacologic vitreolysis with microplasmin increases vitreous diffusion coefficients. Graefes Arch Clin Exp Ophthalmol. 2007;245(4):576-580.

69. Quiram PA, Leverenz VR, Baker RM, Dang L, Giblin FJ, Trese MT. Microplasmin-induced posterior vitreous detachment affects vitreous oxygen levels. Retina. 2007;27(8):1090-1096.

\section{Dovepress}

The manuscript management system is completely online and includes a very quick and fair peer-review system, which is all easy to use. Visit http://www.dovepress.com/testimonials.php to read real quotes from published authors. 